\title{
Kinematic Analysis of A 3-DOF Parallel Mechanism for Milling Applications
}

\author{
T. A. Hess-Coelho ${ }^{*}$, G.E.E. Gojtan and G. P. Furtado \\ Department of Mechatronics and Mechancal Systems Engineering, University of Sao Paulo, São Paulo, Brazil
}

\begin{abstract}
Parallel mechanisms have been studied during the last two decades, due to the fact that they present some potential advantages in a comparison with serial structures. This work presents the kinematic analysis of a novel 3-dof asymmetric parallel mechanism, purposely conceived for milling applications. In a comparison with the previous proposed concepts, this type of kinematic structure shows some advantages. The architecture is simpler and lighter than Tricept because it has no central passive limb. In addition, only the central active limb needs to satisfy the parallelism and orthogonality conditions. Furthermore, one degree of freedom, associated to the third actuator, is decoupled from the other two. Important issues, related to this type of kinematic structure, such as position and velocity analysis, singularity occurrence prediction and workspace evaluation, are discussed in detail.
\end{abstract}

Keywords: Kinematics, parallel mechanisms, milling.

\section{INTRODUCTION}

Parallel mechanisms have been studied during the last two decades, due to the fact that they present some potential advantages in a comparison with serial structures, namely, the ability to perform fast motions, modular construction, lightness and high load capacity [1-3]. In fact, this superiority has been observed, for instance, in pick-and-place parallel robots, capable of reaching very high speeds and accelerations [4].

Regarding the utilization of parallel mechanisms on milling applications, a great number of architectures have been investigated. The first parallel milling machines, employed for this purpose, like the Variax and Hexaglide $[1,5]$, had six degrees of freedom. However, such structures present some drawbacks, namely, the small ratio of workspace volume to machine size and the need to control six axes to position the cutting tool. On the other hand, the Neos Tricept [6] presents a hybrid architecture, where the parallel portion is a tetrapod with one central passive limb to constrain the motion of the moving platform. Differently of its predecessors, this machine has been used in automotive and aerospace industry. Recently, there is a trend for using parallel mechanisms with simpler structures, such as the 3degree-of-freedom Universal Cartesian Robot [7], Tripteron [8] and Orthoglide [9].

Some architectures, as those analyzed in $[7,8,10]$, have reduced modeling complexity since their kinematic equations are linear and fully decoupled. However, due to the fact that these robots are overconstrained mechanisms, they require a very special care on manufacturing and assembly of their parts. Such requirements often demand more tight dimensional and geometrical tolerances, increasing their costs.

*Address correspondence to this author at the Department of Mechatronics and Mechancal Systems Engineering, University of Sao Paulo, São Paulo, Brazil; Tel: +55-11-3091-5760; Fax: 55-11-3091-5461; E-mail: tarcisio.coelho@poli.usp.br
Furthermore, most of the proposed parallel robot architectures present symmetric kinematic chains, while there are only few works dealing with asymmetric architectures [1114]. This fact reflects the preference of researchers for a modular structure, seeking a behavior as close as possible to the isotropic. However, there are some applications, such as machining, in which the speed and rigidity requirements do not need to be the same in all directions.

Another important remark is that, despite the number of proposed parallel robot structures, they have not reached either an acceptable ratio of workspace volume to machine size, or even the precision level required for milling machines yet.

This paper introduces a novel 3-dof parallel mechanism, the $2 U \underline{P} S+\underline{P} R P$, purposely conceived for milling applications. The section 2 deals with the chosen structural synthesis procedure, while other important issues such as the kinematic analysis, singular configurations and workspace are presented in sections 3,4 and 5, respectively.

\section{STRUCTURAL SYNTHESIS}

The structural synthesis goal is to generate a parallel mechanism, able to position the cutting tool in the 3D-space, in such a way that it performs only three independent translations. In this work, an alternative type synthesis procedure, proposed by Hess-Coelho [15] and cited in [16], is employed. Basically, the procedure has three steps: structural synthesis of the mechanism by the method of addition of passive limb; then, the elimination of one among the other active limbs; finally, setting active the constraining passive limb.

According to the first step, to constrain the tool motions, we choose the kinematic chain PRP as the passive limb (Fig. 1a). Then, one feasible mechanism for the task is the 3 $U \underline{P} S+\underline{P} R P$ (Fig. 1b). In fact, the peripheral limbs, whose connectivities $-C_{1}, C_{2}$ and $C_{3}$ - equal 6 , do not restrict the 


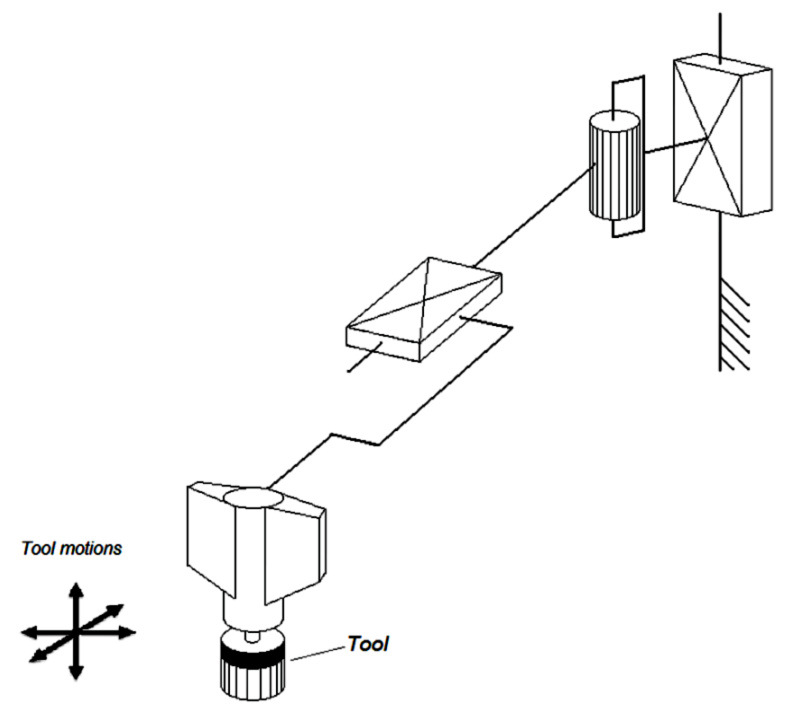

(a)

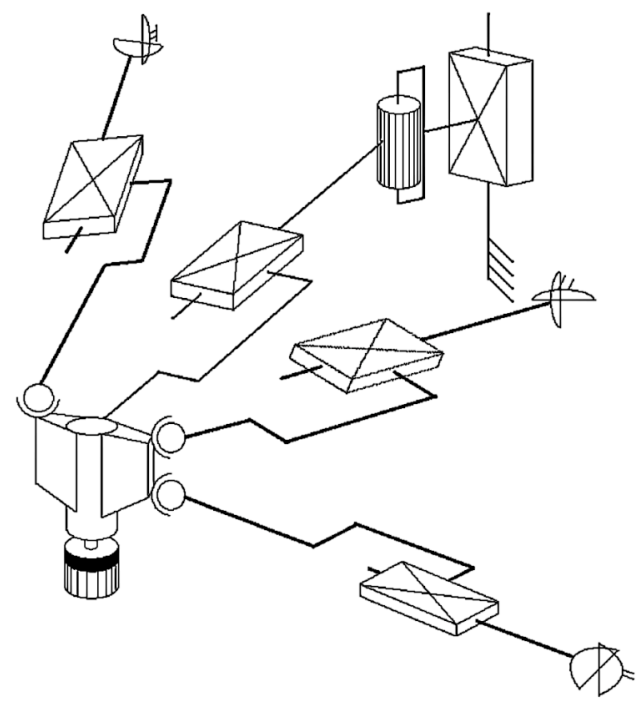

(b)

Fig. (1). Structural synthesis procedure: (a) the constraining limb, (b) the $3 U \underline{P} S+P R P$.
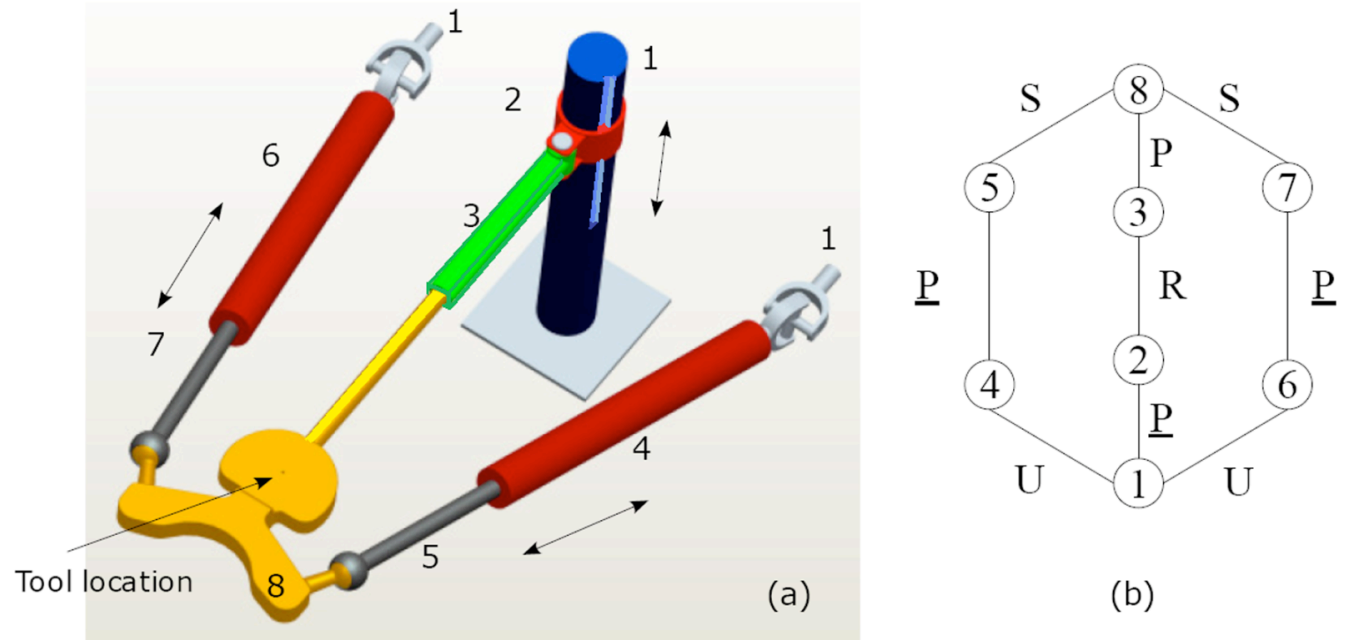

(b)

Fig. (2). The $2 U \underline{P} S+\underline{P} R P$ : (a) the CAD model, (b) the graph representation.

tool movement. By applying the second step, we eliminate one active $U \underline{P} S$ limb. Finally, we set active the constraining limb by coupling an actuator to drive the prismatic joint closest to the base. Fig. (2) indicates the synthesized parallel mechanism, the $2 U \underline{P} S+\underline{P} R P$.

The $2 U \underline{P} S+\underline{P} R P$ corresponds to an asymmetric kinematic structure, once their active limbs are not identical. In addition, the chosen central limb constrains the motions of the tool to remain inside a cylindrical workspace. In accordance with the Kutzbach-Gruebler criterion [2], $\lambda=6, n=8$, $\mathrm{j}=9, \mathrm{j}_{1}=5, \mathrm{j}_{2}=\mathrm{j}_{3}=2$, and the mobility equation yields $\mathrm{M}=6(8-1-$ $9)+1 \times 5+2 \times 2+3 \times 2=3$. Hence, the mechanism has three degrees of freedom.

Constructively, one feasible actuation scheme might utilize servo eletric motors, coupled to ball-screw drives, in such a way to set active the prismatic joints of the parallel mechanism. Other possible architectures, generated by the employed procedure, could be the $2 \underline{P} U S+\underline{P R P}$ and the
$2 \underline{R} U S+\underline{P} R P$. However, the topology of the last one would be more appropriate for manipulation and less indicated for milling applications.

\section{KINEMATIC MODELING AND ANALYSIS}

In oder to describe the motion of the cutting tool, two reference frames are chosen: one in the fixed frame $\mathrm{O} \mathrm{x}_{\mathrm{b}} \mathrm{y}_{\mathrm{b}} \mathrm{Z}_{\mathrm{b}}$ and another in the moving platform $M \boldsymbol{\rho} \boldsymbol{\tau} \boldsymbol{k}$. Regarding the tool position variables, instead of selecting the cartesian coordinates of point $\mathrm{P},\left[\mathrm{x}_{\mathrm{P}}, \mathrm{y}_{\mathrm{P}}, \mathrm{z}_{\mathrm{P}}\right]^{\mathrm{T}}$, we prefer the following cylindrical coordinates $\mathrm{R}, \theta$ and $\mathrm{H}$ (Fig. 3). For the displacements provided by the three actuators, we describe them by using the variables $h_{1}, h_{2}$ and $h_{3}$ (Fig. 4). The symbols $l_{u}, l_{s}, \mathrm{~L}$ and $\mathrm{r}$ represent the structural parameters, which are associated to the relative position of points $\mathrm{U}_{1}, \mathrm{U}_{2}$, $\mathrm{S}_{1}, \mathrm{~S}_{2}$ and $\mathrm{P}$ with respect to the moving platform and base frames in accordance with Tables $\mathbf{1}$ and $\mathbf{2}$. 
Table 1. Coordinates of the Centers of the Universal Joints (Base Frame)

\begin{tabular}{|c|c|c|c|}
\hline & $\mathbf{x}$ & $\mathbf{y}$ & $\mathbf{z}$ \\
\hline \hline${ }^{b} U_{1}$ & 0 & $l_{u}$ & 0 \\
\hline${ }^{b} U_{2}$ & 0 & $-l_{u}$ & 0 \\
\hline
\end{tabular}

Table 2. Coordinates of Points $S_{1}, S_{2}$ and $P$ (Moving Plataform Frame)

\begin{tabular}{|c|c|c|c|}
\hline & $\boldsymbol{\rho}$ & $\boldsymbol{\tau}$ & $\mathbf{k}$ \\
\hline \hline${ }^{m} S_{1}$ & 0 & $l_{S}$ & 0 \\
\hline${ }^{m} S_{2}$ & 0 & $-l_{S}$ & 0 \\
\hline${ }^{m} P$ & $-\mathrm{r}$ & 0 & 0 \\
\hline
\end{tabular}

\section{Position Analysis}

In this work, the first phase of the position analysis deals with the process of generating a null vector of mathematical functions $f_{3 x I}$ that express the dependency between the tool coordinates and the displacements provided by the actuators. Then, the second phase will treat of the solution of the correspondent system of nonlinear equations, widely known as the inverse and the direct problem [2].

The coordinates of point $\mathrm{P}$ are

$\left[\begin{array}{c}b \\ 1\end{array}\right]=\left[\begin{array}{c}b \\ { }^{b} x_{P} \\ b \\ y_{P} \\ { }^{b} z_{P} \\ 1\end{array}\right]=\operatorname{Trans}\left(H, z_{b}\right) \operatorname{Trans}\left(L, x_{b}\right) \operatorname{Rot}\left(\theta, z_{b}\right) \operatorname{Trans}(R-r, \boldsymbol{\rho})\left[\begin{array}{c}m \\ 1\end{array}\right]$

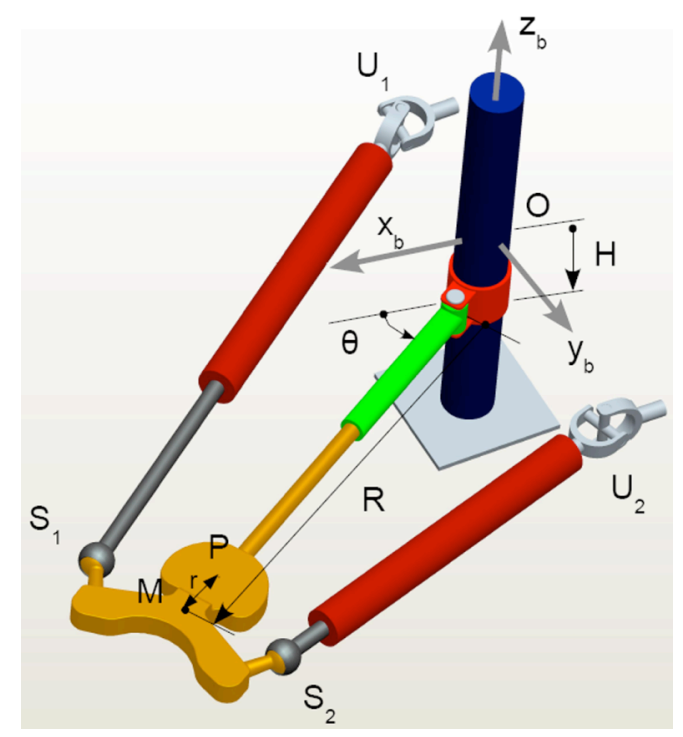

Fig. (3). Cutting tool coordinates, centers of the universal and spherical joints, base frame.

In addition, the coordinates of points $S_{j}(j=1,2)$, the centers of the spherical joints, are

$$
\left[\begin{array}{c}
{ }^{b} S_{j} \\
1
\end{array}\right]=\operatorname{Trans}\left(H, z_{b}\right) \operatorname{Trans}\left(L, x_{b}\right) \operatorname{Rot}\left(\theta, z_{b}\right) \operatorname{Trans}(R, \boldsymbol{\rho})\left[\begin{array}{c}
{ }^{m} S_{j} \\
1
\end{array}\right]
$$

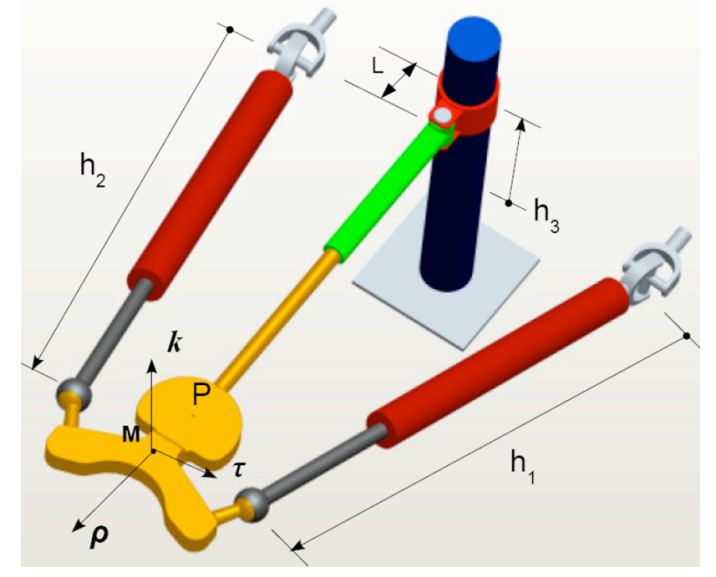

Fig. (4). Displacements provided by the actuators and the moving platform frame.

The necessary equations that couple the variables $R, \theta$ and $H$ with $h_{1}, h_{2}$ and $h_{3}$ are the following:

$$
\begin{gathered}
\left({ }^{b} S_{j}-{ }^{b} U_{j}\right)^{T}\left({ }^{b} S_{j}-{ }^{b} U_{j}\right)=h_{j}{ }^{2} \quad(j=1,2) \\
H=h_{3}
\end{gathered}
$$

Consequently, the null vector $\boldsymbol{f}(P, \boldsymbol{q}, \boldsymbol{\Pi})=\left[f_{1}, f_{2}, f_{3}\right]^{\mathrm{T}}=\mathbf{0}$ can be determined

$$
\begin{aligned}
& f_{1}\left(R, \theta, H, h_{1}, h_{2}, h_{3}\right)=R^{2}+l_{s}^{2}+l_{u}^{2}+H^{2}-2\left(R \sin \theta+l_{s} \cos \theta\right)-h_{1}^{2}=0 \\
& f_{2}\left(R, \theta, H, h_{1,} h_{2,} h_{3}\right)=R^{2}+l_{s}^{2}+l_{u}^{2}+H^{2}+2\left(R \sin \theta-l_{s} \cos \theta\right)-h_{2}^{2}=0 \\
& f_{3}\left(R, \theta, H, h_{1}, h_{2}, h_{3}\right)=H-h_{3}=0
\end{aligned}
$$

Considering the second phase of the position analysis, specifically the inverse problem, the variables $R, \theta$ and $H$ are known and, according to Eq. (5-7), $h_{1}, h_{2}, h_{3}$ can be calculated in a very straightforward manner.

On the other hand, for the direct problem, only the variables $h_{1}, h_{2}, h_{3}$ are known. From Eq. (7), one can obtain $H$ because it coincides with $h_{3}$. In order to determine $R$, we can eliminate the variable $\theta$ from Eq. $(5,6)$, which leads to the following 6 th-degree-polynomial equation

$$
\begin{aligned}
& 4 R^{6}+8 M R^{4}+\left(4 M^{2}-16 Q\right) R^{2}+N=0 \\
& M=h_{3}^{2}+l_{s}^{2}+l_{u}^{2}-\frac{\left(h_{1}^{2}+h_{2}^{2}\right)}{2}, \\
& N=\left(h_{1}^{2}-h_{2}^{2}\right)^{2} l_{s}^{2} \quad Q=l_{s}^{2} l_{u}^{2}
\end{aligned}
$$

If we consider only the positive values for $R$, the direct problem will have at most three solutions. The variable $\theta$ can be calculated from Eq. $(9,10)$.

$$
\begin{aligned}
& \sin \theta=\frac{h_{2}^{2}-h_{1}^{2}}{4 R l_{u}} \\
& \cos \theta=\frac{2\left(R^{2}+l_{s}^{2}+H^{2}+l_{u}^{2}\right)-h_{1}^{2}-h_{2}^{2}}{4 l_{s} l_{u}}
\end{aligned}
$$

\section{Velocity Analysis}

By deriving the position equations with respect to time, one can obtain the mathematical relations between the endeffector velocity vector $\left[\begin{array}{lll}\dot{\theta} & \dot{R} & \dot{H}\end{array}\right]^{T}$ and the actuators linear 
velocities $\left[\begin{array}{lll}\dot{h}_{1} & \dot{h}_{2} & \dot{h}_{3}\end{array}\right]^{T}$. Eq. (11) becomes important for two reasons: the motion planning of the end-effector path and the prediction of singular configurations.

$$
\left[\begin{array}{ccc}
J_{p_{11}} & J_{p_{12}} & J_{p_{13}} \\
J_{p_{21}} & J_{p_{22}} & J_{p_{23}} \\
0 & 0 & 1
\end{array}\right]\left[\begin{array}{c}
\dot{\theta} \\
\dot{R} \\
\dot{H}
\end{array}\right]=\left[\begin{array}{ccc}
h_{1} & 0 & 0 \\
0 & h_{2} & 0 \\
0 & 0 & 1
\end{array}\right]\left[\begin{array}{c}
\dot{h_{1}} \\
\dot{h_{2}} \\
\dot{h_{3}}
\end{array}\right]
$$

where

$$
\begin{aligned}
& J_{p_{11}}=\left(-R c \theta+l_{s} s \theta\right) l_{u}, \quad J_{p_{21}}=\left(R c \theta+l_{s} s \theta\right) l_{u} \\
& J_{p_{12}}=R-l_{u} s \theta, \quad J_{p_{12}}=R+l_{u} s \theta, \quad J_{p_{13}}=H, J_{p_{13}}=H
\end{aligned}
$$

\section{SINGULAR CONFIGURATIONS}

At singular configurations, a parallel mechanism may become stiffer or even uncontrollable. In order to predict such undesirable behaviors, we adopt jacobian analysis as a valid procedure for determining the occurrence of singularities. Starting by the inspection of the jacobian matrix $J_{h}$, we determined three different conditions for annulling its determinant: when either $h_{l}=0$ (Fig. 5a), $h_{2}=0$ (Fig. 5b) or even both are null (Fig. 5c). Fortunately, due to constructive issues, these conditions are not feasible.

Continuing with the singularities investigation, the expression of the determinant of the jacobian matrix $J_{p}$ is,

$$
\operatorname{det} J_{p}=2 l_{u}\left(-R^{2} c \theta+l_{u} l_{s} s^{2} \theta\right)
$$

Then, by varying the angle $\theta$ from - $\pi / 2$ to $\pi / 2$, we can calculate the correspondent values of variable $R$ for annulling det $J_{p}$. Hence, the plot of the singularity locus can be generated as function of the $\mathrm{x}$ and $\mathrm{y}$ coordinates of point $\mathrm{P}$, for a given set of design parameters (see section 5).

\section{Geometric Interpretation}

Despite the fact that the expression of the determinant of $J_{p}$ is available and has low mathematical complexity, it is not an easy task to identify the correspondent mechanism configuration for each position of point P. Consequently, in this section, we also develop an alternative form of this determinant in order to provide a geometric interpretation to the reader.

In Fig. (6), the variable $h_{l}$ corresponds to the distance between the points $S_{l}$ and $U_{l}$. The angles $\alpha_{l}$ and $\gamma_{1}$ define the orientation of the vector $\left(S_{l}-U_{l}\right)$. Similar notation is also employed for the other variables used in the second peripheral limb.

In accordance with the employed notation in Fig. (6), the expressions of the vectors $\left(S_{1}-U_{1}\right)$ and $\left(S_{2}-U_{2}\right)$ are,

$$
\begin{aligned}
& \left({ }^{b} S_{1}-{ }^{b} U_{1}\right)=h_{1} \quad\left(\cos \gamma_{1} \cos \alpha_{1} \rho+\cos \gamma_{1} \sin \alpha_{1} \tau+\sin \gamma_{1} k\right) \\
& \left({ }^{b} S_{2}-{ }^{b} U_{2}\right)=h_{2} \quad\left(\cos \gamma_{2} \cos \alpha_{2} \rho+\cos \gamma_{2} \sin \alpha_{2} \tau+\sin \gamma_{2} \boldsymbol{k}\right)
\end{aligned}
$$

The velocity vectors $\boldsymbol{V}_{\boldsymbol{s} \mathbf{1}}$ and $\boldsymbol{V}_{\boldsymbol{s} \mathbf{2}}$, as functions of $\dot{R}, \dot{\theta}$ and $\dot{H}$, can be obtained as follows,

$$
\begin{aligned}
& \boldsymbol{V}_{S 1}-\boldsymbol{V}_{U 1}=\boldsymbol{V}_{S 1}=\left(\dot{R}-l_{s} \dot{\theta}\right) \rho+R \quad \dot{\theta} \boldsymbol{\tau}+H \boldsymbol{k} \\
& V_{S 2}-V_{U 2}=V_{S 2}=\left(\dot{R}+l_{s} \dot{\theta}\right) \rho+R \quad \dot{\theta} \tau+H \boldsymbol{k}
\end{aligned}
$$
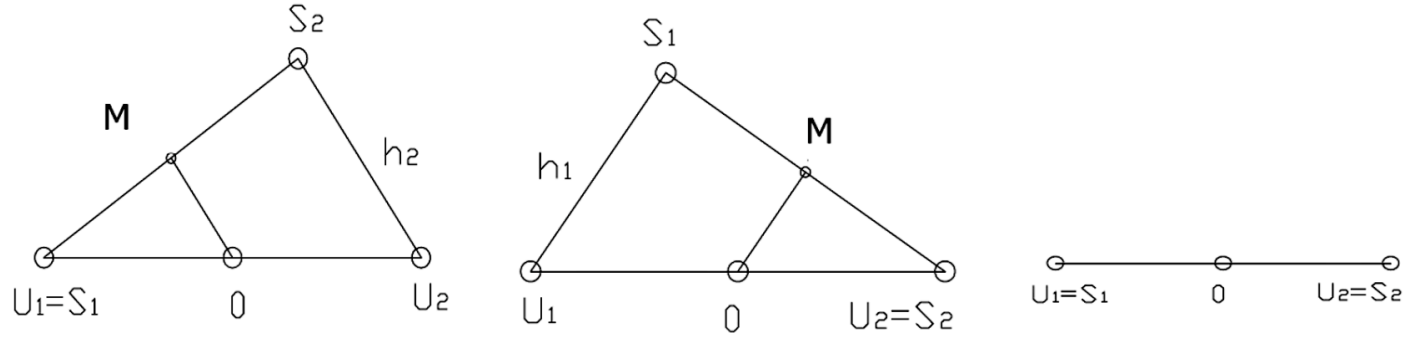

Fig. (5). Singular configurations when $\operatorname{det} J_{h}$ is null: (a) $\mathrm{h}_{1}=0$, (b) $\mathrm{h}_{2}=0$, (c) or both.

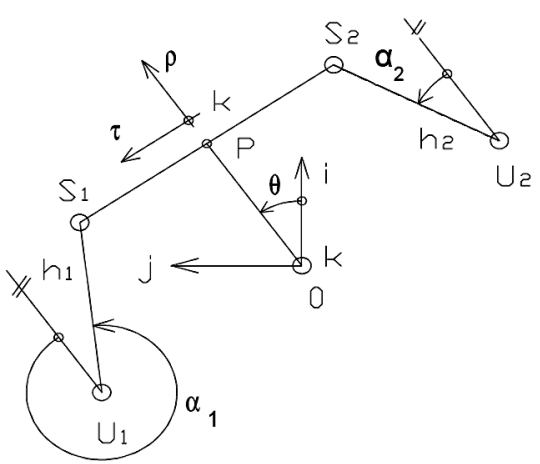

(a)

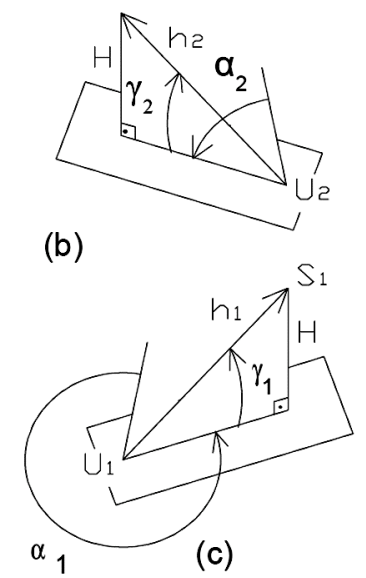

Fig. (6). Kinematic modeling for the alternative formulation to obtain the determinant of Jp:(a) top view of plane $\rho \tau$; $(\mathbf{b})$ vector $\left(\mathrm{S}_{2}-\mathrm{U}_{2}\right)$; $(\mathbf{c})$ vector $\left(\mathrm{S}_{1}-\mathrm{U}_{1}\right)$. 
once,

${ }^{b} V_{S_{1}}{ }^{T}\left({ }^{b} S_{1}-{ }^{b} U_{1}\right)=h_{1} \cdot \dot{h}_{1}$

${ }^{b} V_{S_{2}}{ }^{T}\left({ }^{b} S_{2}-{ }^{b} U_{2}\right)=h_{2} \cdot \dot{h_{2}}$

By replacing the expressions of the vectors from eq.(13$16)$ in eq. $(17,18)$, and by adding the identity $\dot{\boldsymbol{H}}=\dot{\boldsymbol{h}}_{3}$, we can obtain

$$
\left[\begin{array}{ccc}
J_{p_{11}} & J_{p_{12}} & J_{p_{13}} \\
J_{p_{21}} & J_{p_{22}} & J_{p_{23}} \\
0 & 0 & 1
\end{array}\right]\left[\begin{array}{c}
\dot{\theta} \\
\dot{R} \\
\dot{H}
\end{array}\right]=\left[\begin{array}{ccc}
h_{1} & 0 & 0 \\
0 & h_{2} & 0 \\
0 & 0 & 1
\end{array}\right]\left[\begin{array}{c}
\dot{h}_{1} \\
\dot{h_{2}} \\
\dot{h_{3}}
\end{array}\right]
$$

where

$$
\begin{aligned}
& J_{p_{11}}=h_{1}\left(-l_{s} \cos \gamma_{1} \cos \alpha_{1}+R \cos \gamma_{1} \sin \alpha_{1}\right) \\
& J_{p_{21}}=h_{2}\left(l_{s} \cos \gamma_{2} \cos \alpha_{2}+R \cos \gamma_{2} \sin \alpha_{2}\right) \\
& J_{p_{12}}=h_{1} \cos \gamma_{1} \cos \alpha_{1} \quad J_{p_{22}}=h_{2} \cos \gamma_{2} \cos \alpha_{2} \\
& J_{p_{13}}=H \quad J_{p_{23}}=H
\end{aligned}
$$

To determine the conditions for annulling det $J_{p}$ (Eq. 19), we assume that both $\gamma_{1}$ and $\gamma_{2}$ are different from $\pm \pi / 2$. In addition, we do not consider here the cases when either $h_{l}$, $h_{2}$ or even both are null.

$$
\operatorname{det} J_{p}=h_{1} h_{2} c \gamma_{1} c \gamma_{2}\left(-2 l_{s} c \alpha_{1} c \alpha_{2}+R \sin \left(\alpha_{1}-\alpha_{2}\right)\right)
$$

Then, two cases need to be taken into account: (a) when $\alpha_{1}$ and $\alpha_{2}$ are equal; (b) when $\alpha_{1}=\alpha_{2}$. By analyzing the first case, one can obtain that $\alpha_{1}=\alpha_{2}= \pm \pi / 2$. For the second case, the analysis leads to two results: (b1) $\alpha_{1}=\alpha_{2}= \pm \pi / 2$; (b2) $\tan \alpha_{1}=l_{s} R^{-1}$ and $\tan \alpha_{2}=-l_{s} R^{-1}$. By examining the last two equations (for "b2"), one can notice that they mean that the center of the universal joints $U_{1}$ and $U_{2}$ are coincident with the center of the revolute joint $\mathrm{O}$. Fortunately, also due to constructive issues, this condition is not feasible.

Fig. (7) shows the case when $\alpha_{1}=-\alpha_{2}=-\pi / 2$, while the angles $\gamma_{1}$ and $\gamma_{2}$ are not null. For this case, the points $U_{1}, \mathrm{~S}_{1}$, $S_{2}$ and $U_{2}$ belong to the same vertical plane. This configuration is certainly singular and must be avoided. The main reason is that, from such configuration, the moving platform of the parallel mechanism can only move in the $\boldsymbol{k}$ direction, but it is not able to change its position in the $\boldsymbol{\rho}$ and $\boldsymbol{\tau}$ directions.

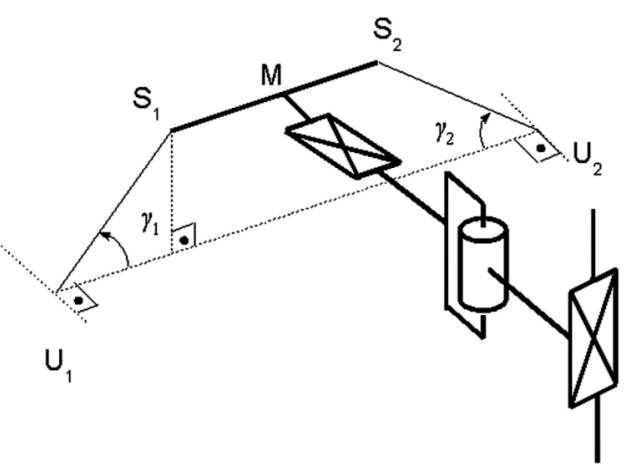

Fig. (7). An example of singular configuration when $\operatorname{det} J_{p}$ is null: points $U_{1}, \mathrm{~S}_{1}, S_{2}$ and $U_{2}$ belong to the same vertical plane.

\section{WORKSPACE}

The available workspace of the $2 U P S+P R P$ represents a $3 \mathrm{D}$-region where the cutting tool tip, that belongs to the moving platform, can move. To determine this workspace, the discretization method [13] is employed. This method considers that the workspace is determined from a solid, assumed larger than the feasible workspace, discretized by a regular mesh. Then, a procedure checks whether or not each mesh node violates the physical and kinematic constraints. Consequently, workspace boundaries are composed by a set of nodes that has at least one neighbor node that does not belong to the workspace.

The physical constraints are represented by both the actuators strokes and the passive joints limits. In addition, another important issue refers to the kinematic constraints. Our analysis calculates the determinants of Jacobian matrices $J_{h}$ and $J_{P}$ and verifies if their values are null, which correspond to singular configurations (Figs. 8 and 9). Table 3 presents the geometric parameters and the actuators strokes for the parallel mechanism. Table 4 shows the values of the passive joints limits.

Table 3. Geometric Parameters and the Actuators Strokes for the Parallel Mechanism

\begin{tabular}{|c|c|c|}
\hline Symbol & Description & $\begin{array}{c}\text { Value } \\
\text { [mm] }\end{array}$ \\
\hline \hline$L$ & $\begin{array}{c}\text { Position of the revolute joint center, in the x- } \\
\text { direction, with respect to the base frame }\end{array}$ & 125 \\
\hline$l_{u}$ & $\begin{array}{c}\text { Position of the center of the universal joints, } \\
\text { with respect to the base frame }\end{array}$ & 400 \\
\hline$l_{s}$ & $\begin{array}{c}\text { Position of the center of the spherical joints, } \\
\text { with respect to the platform frame }\end{array}$ & 200 \\
\hline$r$ & $\begin{array}{c}\text { Position of point P, in x-direction, with respect } \\
\text { to the platform frame }\end{array}$ & 100 \\
\hline$H$ & Stroke of the prismatic joint actuator at the \\
central limb & -600 to \\
\hline
\end{tabular}

Table 4. Passive Joints Limits

\begin{tabular}{|c|c|c|c|c|}
\hline Symbol & Description & $\begin{array}{c}\text { Limits } \\
{[\mathbf{d e g}]}\end{array}$ & $\begin{array}{c}\mathbf{L}_{\mathbf{m i n}} \\
{[\mathbf{m m}]}\end{array}$ & $\begin{array}{c}\mathbf{L}_{\mathbf{m a x}} \\
{[\mathbf{m m}]}\end{array}$ \\
\hline \hline$\beta_{1,2}$ & Universal joint limits & \pm 45 & - & - \\
\hline$\varphi$ & Spherical joint limits & \pm 30 & - & - \\
\hline$\Theta$ & Revolute joint limits & \pm 34 & & 1295 \\
\hline$h_{1,2}$ & $\begin{array}{c}\text { Min. and max. strokes for } \\
\text { actuators 1 and 2 }\end{array}$ & - & 850 & 1053 \\
\hline$h_{3}$ & $\begin{array}{c}\text { Min. and max. strokes for the } \\
\text { actuator 3 }\end{array}$ & - & 604 & 1400 \\
\hline$R$ & $\begin{array}{r}\text { Prismatic joint limits at the } \\
\text { central limb }\end{array}$ & & 600 & 1200 \\
\hline
\end{tabular}




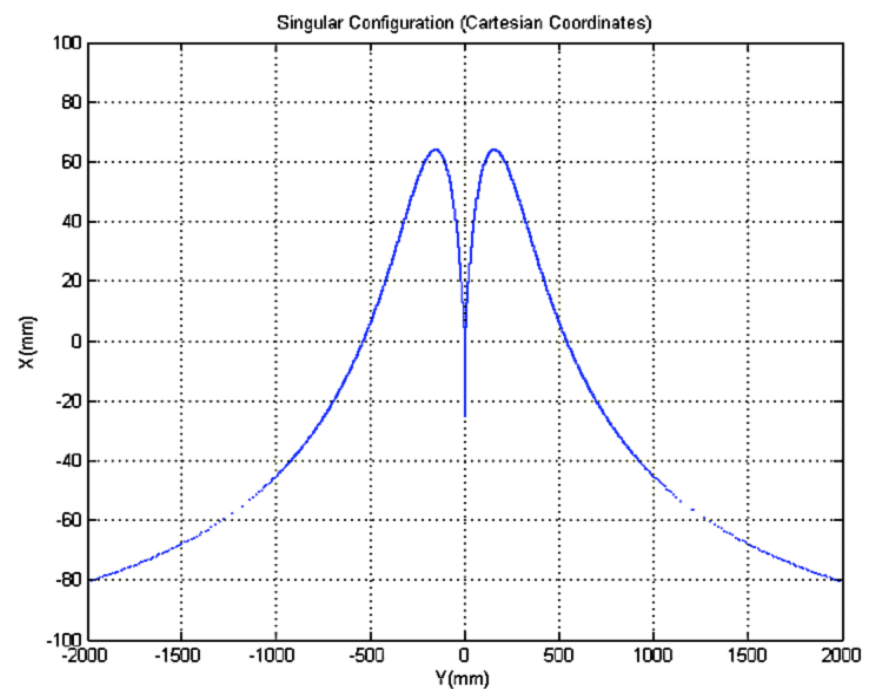

Fig. (8). Singularity locus when det $J_{p}$ is null.

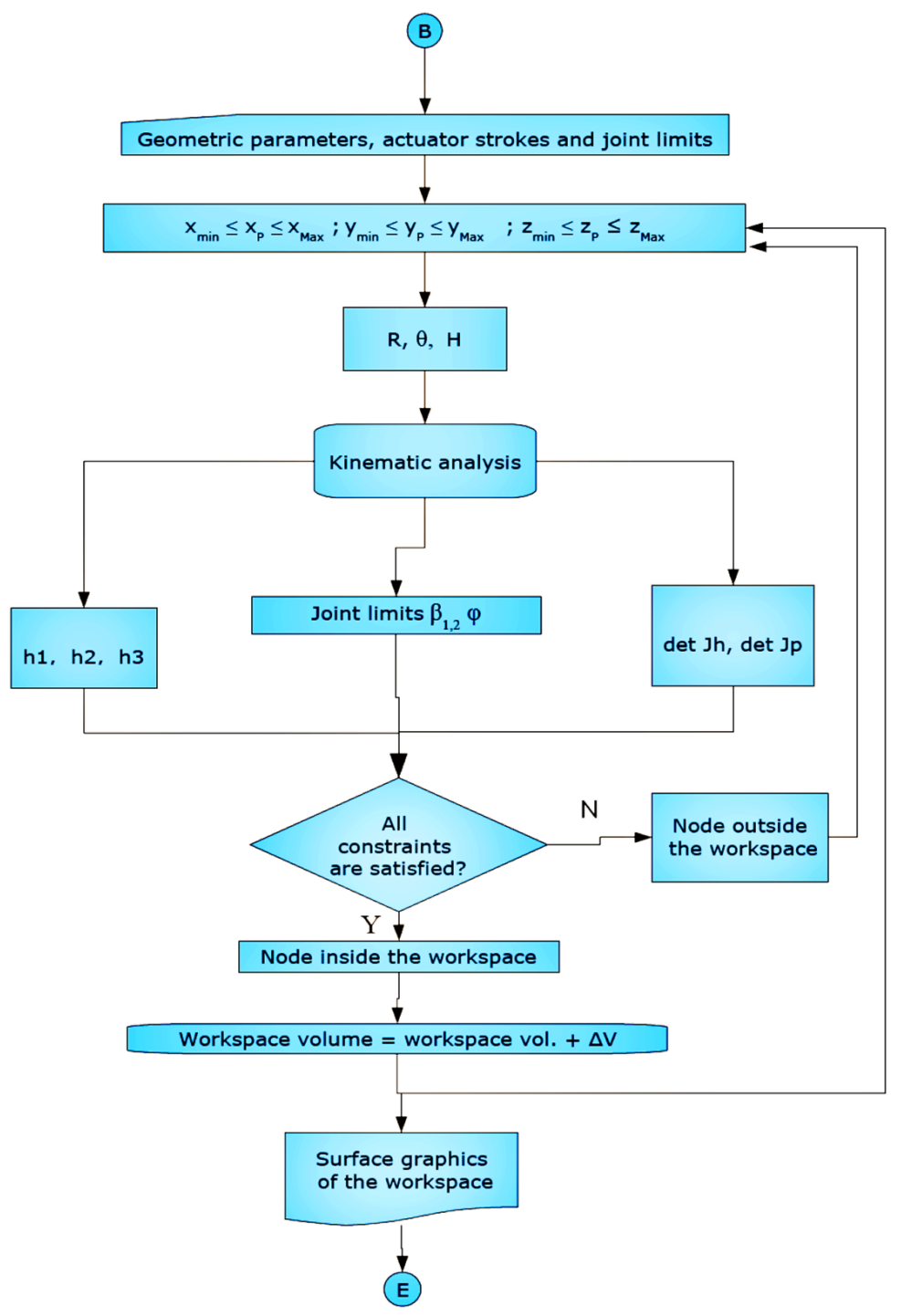

Fig. (9). Flow chart for the computation of the workspace volume and the generation of the surface graphics.

The volume of the available workspace is approximately $216 \mathrm{dm}^{3}$ and Fig. (10) presents the shape of the obtained workspace at different heights $\mathrm{H}$, from 0 to $600 \mathrm{~mm}$.

\section{CONCLUSIONS}

This paper introduced a novel 3-dof asymmetric and parallel mechanism, the $2 U \underline{P} S+\underline{P} R P$, purposely conceived 


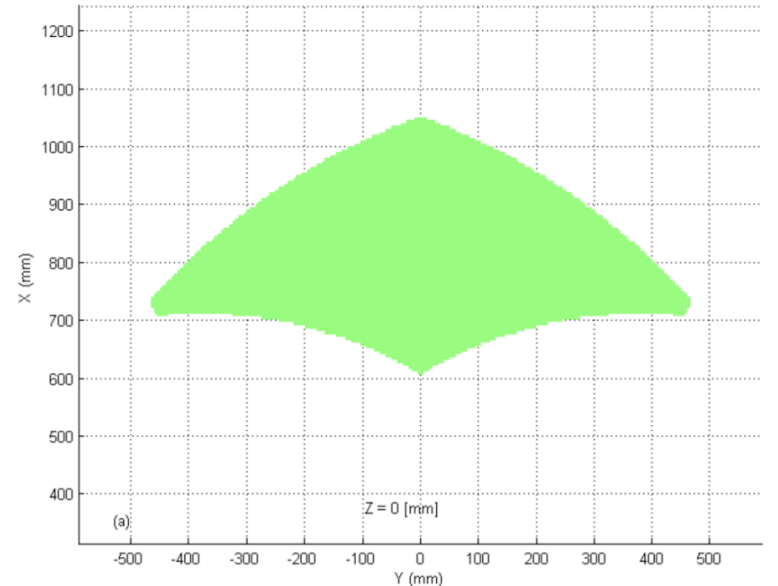

(a)

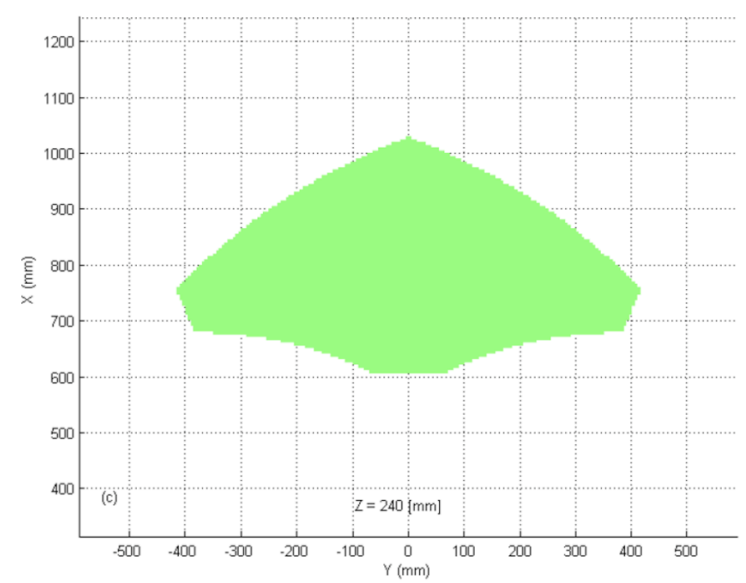

(c)

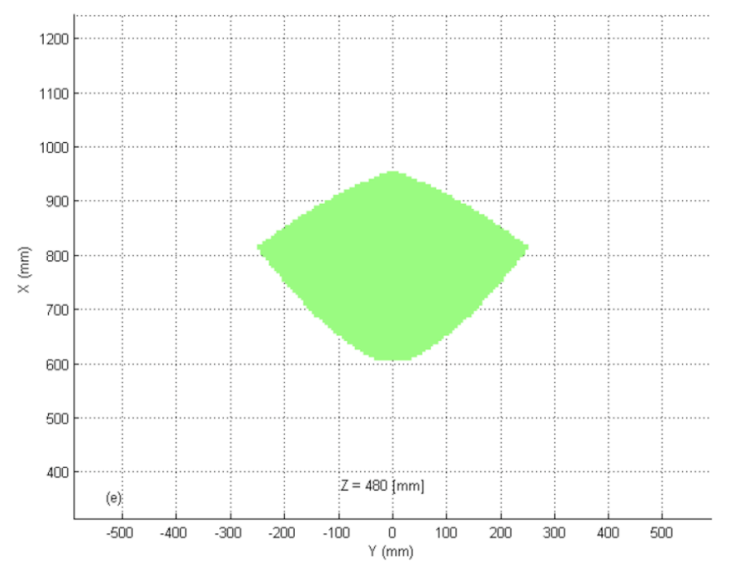

(e)

Fig. (10). Shape of the obtained workspace for: (a) $H=0 \mathrm{~mm}$; (b) $H=120 \mathrm{~mm}$; (c) $H=240 \mathrm{~mm}$; (d) $H=360 \mathrm{~mm}$; (e) $H=480 \mathrm{~mm}$; (f) $H$ $=600 \mathrm{~mm}$.

for milling applications. In a comparison with the previous proposed concepts, this asymmetric and parallel kinematic structure has some advantages. The architecture is simpler and lighter than Tricept because it has no central passive limb. In addition, the $2 U \underline{P S}+\underline{P R P}$ does not need a serial $\underline{R R}$ wrist to guide the cutting tool in order to perform three independent translations. Other proposed concepts, for instance, Orthoglide and Tripteron, need to satisfy special

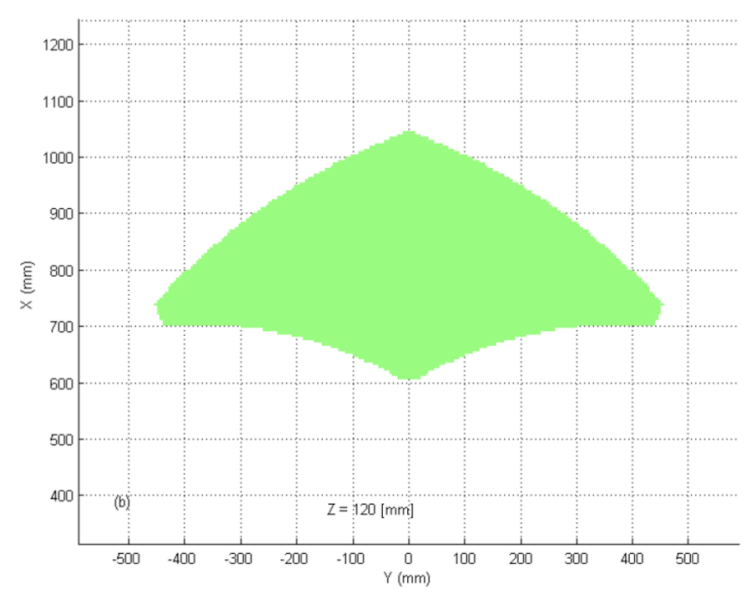

(b)

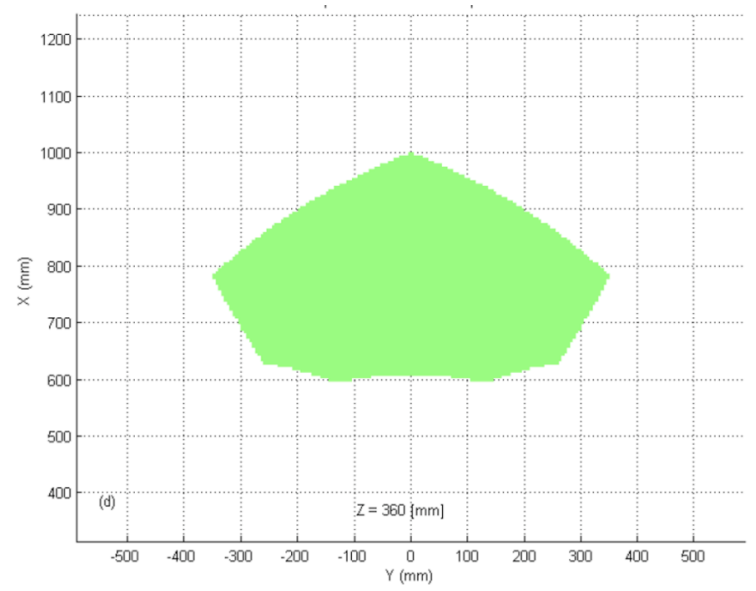

(d)

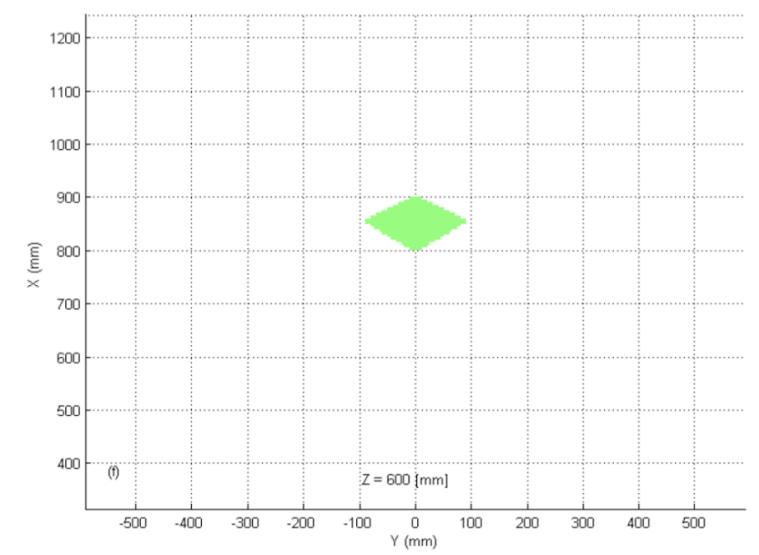

(f)

assembly conditions: the orthogonality and the parallelism between the axes of passive and active joints in their three limbs. For the $2 U \underline{P S}+\underline{P R P}$, such special conditions refer only to the central limb, which might reduce its manufacturing and assembly costs.

As a consequence of this type of parallel mechanism, one degree of freedom, associated to the third actuator, is 
decoupled from the other two. Such feature brings some benefits on the kinematic model, namely, the low complexity of the position kinematics (both for the inverse and direct problems), the velocity analysis and the prediction of occurrence of singularities, as well.

Regarding the prediction of singularity configurations, two possible formulations were derived. One formulation seems more suitable for plotting the singularity locus, while the other demonstrates to be more convenient for the geometric interpretation. With respect to the singularity locus, when $\operatorname{det} J p$ is null, we found the same curve for any value of the variable $\mathrm{H}$.

Considering the geometric interpretation, the authors noticed that the parallel mechanism cannot reach singularities of the first kind [2] due to constructive issues. However, depending on the parameter values, the parallel mechanism might reach singularity configurations of the second kind, when the moving platform will perform motions in the $\boldsymbol{k}$ direction, but it will not be able to change its position in the $\boldsymbol{\rho}$ and $\boldsymbol{\tau}$ directions. The authors also observed that the parameter $L$ must be positive, at least onefourth of $l_{u}$, in order to avoid their occurrence.

For the workspace evaluation, we employed the discretization method and the estimated volume, for the chosen parameters, is $216 \mathrm{dm}^{3}$. Inside the calculated workspace, there is no singularity of any kind. Futures works will address the accuracy evaluation of this asymmetric parallel mechanism, dealing with kinematic, geometric and static errors.

\section{REFERENCES}

[1] J.P. Merlet, Parallel robots. 2nd ed. Dordrecht: Springer, 2006.

[2] L.W. Tsai, Robot Analysis: The Mechanics of Serial and Parallel Robots. New York: John Wiley \& Sons, 1999.

[3] M. Ceccarelli, Fundamentals of Mechanics of Robotic Manipulation. Dordrecht: Kluwer, 2004.
[4] Adept Technology Inc., "Adept Quattro s 650H”, Livermore, CA, USA. [Online] Available: http://www.adept.com [Accessed April 30, 2010].

[5] R. Khol, "A machine tool built from mathematics", American Machinist, pp. 53-55, 1994

[6] K.E. Neumann, "Next generation Tricept - a true revolution in parallel kinematics", Parallel Kinematic Machines in Research and Practice. R. Neugebauer, Ed. Zwickau: Verlag Wissenschaftliche Scripten, 2004, vol. 24, pp. 591-594.

[7] H.S. Kim, and L.-W. Tsai, "Design Optimization of a Cartesian Parallel Manipulator", in ASME DETC Confer., 2002, paper DETC2002/MECH-34301

[8] C.M. Gosselin, X. Kong, S. Foucault, and I.A. Bonev, "A fullydecoupled 3-dof translational parallel mechanism", Paralle Kinematic Machines in Research and Practice. R. Neugebauer, Ed. Zwickau: Verlag Wissenschaftliche Scripten, 2004, vol. 24, pp. 595-610.

[9] D. Chablat, P. Wenger, and S. Staicu, "Dynamics of the Orthoglide parallel robot", UPB Scientific Bulletin, Series D: Mechanical Engineering, vol. 71, pp. 3-16, 2009.

[10] R. Di Gregorio, and V. Parenti-Castelli, "Design of 3-dof parallel manipulators based on dynamic performances", Parallel Kinematic Machines in Research and Practice, R. Neugebauer, Ed. Zwickau: Verlag Wissenschaftliche Scripten, 2004, vol. 24, pp. 385-397.

[11] Q. Li, and Z. Huang, "Type Synthesis of 5-DOF Parallel Manipulators", in IEEE Int. Conf. on Robotics and Automation, 2003, pp. 1203-1208.

[12] T. Huang, M. Li, X. M. Zhao, J.P. Mei, D.G. Chetwynd, and S.J. $\mathrm{Hu}$, "Conceptual Design and Dimensional Synthesis for a 3-DOF Module of the TriVariant - A Novel 5-DOF Reconfigurable Hybrid Robot", IEEE Trans. Rob., vol. 21, pp. 449-456, 2005.

[13] V. D. Kumazawa, T. A. Hess-Coelho, D. Rinaudi, G. Carbone, and M. Ceccarelli, "Kinematic Analysis and Operation Feasibility of a 3-dof Asymmetric Parallel Mechanism", in 20th COBEM, 2009, paper COB09-0744.

[14] J. A. Saglia, N. G. Tsagarakis, J. S. Dai, and D. G. Caldwell, "A high performance redundantly actuated parallel mechanism for ankle rehabilitation", The International Journal of Robotics Research, vol. 28, pp. 1216-1227, 2009.

[15] T. A. Hess-Coelho, "An alternative procedure for type synthesis of parallel mechanisms", in 12th IFToMM World Congress, 2007, paper A612.

[16] G. Gogu, Structural Synthesis of Parallel Robots: Part 2 Translational Topologies with Two and Three Degrees of Freedom, Dordrecht: Kluwer, 2009.

(C) Hess-Coelho et al.; Licensee Bentham Open.

This is an open access article licensed under the terms of the Creative Commons Attribution Non-Commercial License (http: //creativecommons.org/licenses/by$\mathrm{nc} / 3.0 /)$, which permits unrestricted, non-commercial use, distribution and reproduction in any medium, provided the work is properly cited. 